\title{
The Joint Creeping Motion of Three Viscid Liquids in a Plane Layer: A Priori Estimates and Convergence to Steady Flow
}

\author{
V. K. Andreev ${ }^{1,2^{*}}$ and E. N. Cheremnykh ${ }^{1,2^{* * *}}$ \\ ${ }^{1}$ Institute of Computational Modelling, Akademgorodok 50, bld. 44, Krasnoyarsk, 660036 Russia \\ ${ }^{2}$ Siberian Federal University, pr. Svobodnyi 79, Krasnoyarsk, 660041 Russia
}

Received May 16, 2015

\begin{abstract}
We study a partially invariant solution of rank 2 and defect 3 of the equations of a viscid heat-conducting liquid. It is interpreted as a two-dimensional motion of three immiscible liquids in a flat channel bounded by fixed solid walls, the temperature distribution on which is known. From a mathematical point of view, the resulting initial-boundary value problem is a nonlinear inverse problem. Under some assumptions (often valid in practical applications), the problem can be replaced by a linear problem. For the latter we obtain some a priori estimates, find an exact steady solution, and prove that the solution approaches the steady regime as time increases, provided that the temperature on the walls stabilizes.
\end{abstract}

DOI: $10.1134 / \mathrm{S} 1990478916010026$

Keywords: thermocapillarity, a priori estimates, conjugate initial-boundary value problem, asymptotic behavior

As is known, motion begins in a nonuniformly heated liquid. In applications, we often encounter the situations when motion is originated in the two or more liquid media that contact one another along certain interfaces. If the liquids do not mix during their interaction then they form some more or less visible interfaces. The oil-water system is a typical example of this situation. The need for modeling multiphase flows, taking into account the differences in physical and chemical factors, arises in the design of cooling systems and power plants, in studying the growth of crystals and films, or in the aerospace industry [1-4].

Some exact solutions of the equations of Marangoni convection are known [5-7]. One of the first was obtained in [8] which is a steady Poiseuille flow of two immiscible liquids in an oblique channel. As a rule, almost all flows were steady and unidirectional. The stability of these flows was investigated in [9, 10]. As far as the nonsteady thermocapillary flows are concerned, their study have begun rather recently [11, 12].

The problem of thermocapillary convection of two incompressible liquids in a container separated by a closed separation surface was studied in [13]. The time-local unique solvability of the problem was obtained in the Hölder classes of functions. The problem of thermocapillary motion of a drop in the entire three-dimensional space was studied in [14]. Moreover, its unique solvability was established in the Hölder classes with a power-like weight. It turned out that the velocity vector field decreases at infinity in same manner as the initial data and mass forces, whereas the temperature tends to a constant equal to the limit of the initial temperature at infinity.

This article is devoted to the study of solutions of the two-dimensional conjugate boundary value problem which results from the linearization of the Navier-Stokes system of equations supplemented with the heat transfer equation. Motion is initiated by thermocapillary forces applied along two interfaces which cause nonsteady Marangoni convection. Such a convection can prevail under conditions of microgravity or in the movement of thin liquid films.

The aim of the work is to obtain some a priori estimates for the solutions of the above conjugate problem in the space of uniform convergence. The estimates of the solutions are obtained using special identities and the Friedrichs Inequality. The main difficulty is encountered in estimating the pressure gradients which are unknown either.

\footnotetext{
${ }^{*}$ E-mail: andr@icm.krasn.ru

*** E-mail: elena_cher@icm.krasn.ru
} 


\section{STATEMENT OF THE PROBLEM}

The two-dimensional motion of a viscid incompressible heat-conducting liquid is described by the system of equations

$$
\begin{gathered}
u_{t}+u u_{x}+v u_{y}+\frac{1}{\rho} p_{x}=\nu\left(u_{x x}+u_{y y}\right), \\
v_{t}+u v_{x}+v v_{y}+\frac{1}{\rho} p_{y}=\nu\left(v_{x x}+v_{y y}\right) \\
u_{x}+v_{y}=0 \\
\Theta_{t}+u \Theta_{x}+v \Theta_{y}=\chi\left(\Theta_{x x}+\Theta_{y y}\right) .
\end{gathered}
$$

Here $u(x, y, t)$ and $v(x, y, t)$ are the components of the velocity vector, $p(x, y, t)$ is the pressure, $\Theta(x, y, t)$ is the temperature, $\rho$ is the density, $\nu$ is the kinematic viscosity, and $\chi$ is the thermal conductivity of the liquid. The quantities $\rho, \nu$, and $\chi$ are assumed constant.

The system of equations ( 1 ) admits a four-dimensional Lie subalgebra $\left\langle\partial_{x}, \partial_{u}+t \partial_{x}, \partial_{p}, \partial_{\Theta}\right\rangle$ [6]. Its invariants are $t, y$, and $v$, and a partially invariant solution of rank 2 and defect 3 should be sought for in the form $u=u(x, y, t), v=v(y, t), p=p(x, y, t)$, and $\Theta=\Theta(x, y, t)$.

Inserting the exact form of the solution into the first three equations of (1) yields

$$
\begin{array}{ll}
u=w(y, t) x+u_{1}(y, t), & w+v_{y}=0, \quad w_{t}+v w_{y}+w^{2}=f(t)+\nu w_{y y}, \\
\frac{1}{\rho} p=d(y, t)-\frac{f(t)}{2} x^{2}, & d_{y}=\nu v_{y y}-v_{t}-v v_{y}, \quad u_{1 t}+v u_{1 y}+u_{1} w=0
\end{array}
$$

with some function $f(t)$ that is arbitrary so far.

Regarding the temperature field, we assume that it has the form

$$
\Theta=a(y, t) x^{2}+a_{1}(y, t) x+b(y, t) .
$$

As we see below, (3) is in good accord with the conditions on the interfaces.

We assume for simplicity that $u_{1}(y, t) \equiv 0$ and $a_{1}(y, t) \equiv 0$. The latter condition means that the temperature field has an extremum at $x=0$ : a maximum for $a(y, t)<0$ and a minimum for $a(y, t)>0$. Let us apply the solution of the form (2), (3) to describe joint motion of three immiscible liquids in the flat layer $0<y<l_{3}$, considering that the walls $y=0$ and $y=l_{3}$ are solid. Introducing the index $j=1,2,3$, for the liquids and using (2) and (3), we come to the conclusion that, in their domains, the unknowns satisfy the equations

$$
\begin{gathered}
w_{j t}+v_{j} w_{j y}+w_{j}^{2}=\nu_{j} w_{j y y}+f_{j}(t), \\
v_{j t}+v_{j} v_{j y}+d_{j y}=\nu_{j} v_{j y y}, \\
w_{j}+v_{j y}=0 \\
a_{j t}+2 w_{j} a_{j}+v_{j} a_{j y}=\chi_{j} a_{j y y}, \\
b_{j t}+v_{j} b_{j y}=\chi_{j} b_{j y y}+2 \chi_{j} a_{j} .
\end{gathered}
$$

At the interfaces $y=l_{n}(x, t)$ for $n=1,2$, the conditions hold [9]:

$$
\begin{array}{rlrl}
w_{1}\left(l_{1}(x, t), t\right) & =w_{2}\left(l_{1}(x, t), t\right), & & w_{2}\left(l_{2}(x, t), t\right)=w_{3}\left(l_{2}(x, t), t\right), \\
v_{1}\left(l_{1}(x, t), t\right) & =v_{2}\left(l_{1}(x, t), t\right), & & v_{2}\left(l_{2}(x, t), t\right)=v_{3}\left(l_{2}(x, t), t\right), \\
l_{1 t}+x w_{1}\left(l_{1}(x, t), t\right) l_{1 x} & =v_{1}\left(l_{1}(x, t), t\right), & & l_{2 t}+x w_{2}\left(l_{2}(x, t), t\right) l_{2 x}=v_{2}\left(l_{2}(x, t), t\right), \\
a_{1}\left(l_{1}(x, t), t\right) & =a_{2}\left(l_{1}(x, t), t\right), & & a_{2}\left(l_{2}(x, t), t\right)=a_{3}\left(l_{2}(x, t), t\right), \\
k_{1} \frac{\partial a_{1}}{\partial n_{1}}=k_{2} \frac{\partial a_{2}}{\partial n_{1}}, & & k_{2} \frac{\partial a_{2}}{\partial n_{2}}=k_{3} \frac{\partial a_{3}}{\partial n_{2}},
\end{array}
$$


where $k_{j}>0$ are the heat conductivity coefficients and $\mathbf{n}_{n}=\left(1+l_{n x}^{2}\right)^{-1 / 2}\left(-l_{n x}, 1\right)$ are the normals to the lines $y=l_{n}(x, t)$. The dynamic conditions for $y=l_{n}(x, t)$ are as follows [9]:

$$
\begin{aligned}
& \left(p_{1}-p_{2}\right) \mathbf{n}_{1}+\left[2 \rho_{2} \nu_{2} D\left(\mathbf{u}_{2}\right)-2 \rho_{1} \nu_{1} D\left(\mathbf{u}_{1}\right)\right] \mathbf{n}_{1}=2 \sigma_{1}\left(\Theta_{1}\right) K_{1} \mathbf{n}_{1}+\nabla_{11} \sigma_{1}, \\
& \left(p_{2}-p_{3}\right) \mathbf{n}_{2}+\left[2 \rho_{3} \nu_{3} D\left(\mathbf{u}_{3}\right)-2 \rho_{2} \nu_{2} D\left(\mathbf{u}_{2}\right)\right] \mathbf{n}_{2}=2 \sigma_{2}\left(\Theta_{2}\right) K_{2} \mathbf{n}_{2}+\nabla_{11} \sigma_{2} .
\end{aligned}
$$

In (8), $D$ is the strain-rate tensor $\mathbf{u}_{j}=\left(x w_{j}(y, t), v_{j}(y, t)\right)$, whereas $\nabla_{11}=\nabla-(\mathbf{n} \cdot \nabla) \mathbf{n}$ on the righthand side designates the surface gradient; $K_{n}=l_{n x x}\left(1+l_{n x}^{2}\right)^{-3 / 2}$ are the mean curvatures of the interfaces $y=l_{n}(x, t)$; while $\sigma_{1}\left(\Theta_{1}\right)$ and $\sigma_{2}\left(\Theta_{2}\right)$ are the surface tension coefficients depending on temperature. For most of real liquid media, the dependence $\sigma_{n}\left(\Theta_{n}\right)$ is approximated well by the linear function

$$
\sigma_{n}\left(\Theta_{n}\right)=\sigma_{n}^{0}-\varkappa_{n} \Theta_{n}
$$

where $\varkappa_{n}>0$ is the temperature coefficients of the surface tension of the lines $y=l_{n}(x, t)$. They are assumed constant and determined by experimental methods.

The dynamic conditions (8) have a vector form. Projecting them to the tangent directions $\boldsymbol{\tau}_{n}=$ $\left(1+l_{n x}^{2}\right)^{-1 / 2}\left(1, l_{n x}\right)$ and using (9) and (2), we obtain

$$
\begin{aligned}
& {\left[\mu_{2} D\left(\mathbf{u}_{2}\right)-\mu_{1} D\left(\mathbf{u}_{1}\right)\right] \mathbf{n}_{1} \cdot \boldsymbol{\tau}_{1}=-\varkappa_{1} \nabla_{11} \Theta_{1} \cdot \boldsymbol{\tau}_{1},} \\
& {\left[\mu_{3} D\left(\mathbf{u}_{3}\right)-\mu_{2} D\left(\mathbf{u}_{2}\right)\right] \mathbf{n}_{2} \cdot \boldsymbol{\tau}_{2}=-\varkappa_{2} \nabla_{11} \Theta_{2} \cdot \boldsymbol{\tau}_{2},}
\end{aligned}
$$

where $\mu_{j}=\rho_{j} \nu_{j}$ are the dynamic viscosity of the liquids. The projections (8) to the normal $\mathbf{n}_{1,2}$ yield

$$
\begin{array}{r}
\rho_{1} d_{1}\left(l_{1}(x, t), t\right)+\left[\rho_{2} f_{2}(t)-\rho_{1} f_{1}(t)\right] x^{2} / 2-\rho_{2} d_{2}\left(l_{1}(x, t), t\right)+\left[2 \mu_{2} D\left(\mathbf{u}_{2}\right)-2 \mu_{1} D\left(\mathbf{u}_{1}\right)\right] \mathbf{n}_{1} \cdot \mathbf{n}_{1} \\
=\left(\sigma_{1}^{0}-\varkappa_{1}\left[a_{1}\left(l_{1}(x, t), t\right) x^{2}+b_{1}\left(l_{1}(x, t), t\right)\right]\right) l_{1 x x}\left(1+l_{1 x}^{2}\right)^{-3 / 2}, \quad \\
\rho_{2} d_{2}\left(l_{2}(x, t), t\right)+\left[\rho_{3} f_{3}(t)-\rho_{2} f_{2}(t)\right] x^{2} / 2-\rho_{3} d_{3}\left(l_{2}(x, t), t\right)+\left[2 \mu_{3} D\left(\mathbf{u}_{3}\right)-2 \mu_{2} D\left(\mathbf{u}_{2}\right)\right] \mathbf{n}_{2} \cdot \mathbf{n}_{2} \\
=\left(\sigma_{2}^{0}-\varkappa_{2}\left[a_{2}\left(l_{2}(x, t), t\right) x^{2}+b_{2}\left(l_{2}(x, t), t\right)\right]\right) l_{2 x x}\left(1+l_{2 x}^{2}\right)^{-3 / 2} .
\end{array}
$$

The boundary conditions on the solid walls have the form

$$
\begin{aligned}
u_{1}(0, t)=0, \quad u_{3}\left(l_{3}, t\right)=0, & v_{1}(0, t)=0, \quad v_{3}\left(l_{3}, t\right)=0, \\
a_{1}(0, t)=a_{10}(t), & a_{3}\left(l_{3}, t\right)=a_{30}(t)
\end{aligned}
$$

with some given functions $a_{10}(t)$ and $a_{30}(t)$.

The initial conditions for the velocities are zero (we study the properties of the solution of the problem simulating the motion only under the action of thermocapillary forces):

$$
u_{j}(y, 0)=0, \quad v_{j}(y, 0)=0 .
$$

Besides,

$$
l_{n}(x, 0)=l_{n}^{0}(x), \quad a_{j}(y, 0)=a_{j}^{0}(y),
$$

while $a_{j}^{0} \neq 0$ for at least one of the indices $j$ so that the thermocapillary effect starts at $t=0$.

Note several specific features of the formulated problem: This is a nonlinear and inverse problem since the functions $f_{j}(t)$ are the sought-for. It is easy if we eliminate $v_{j}(y, t)$ from the equations of mass conservation. Then the problem reduces to the conjugate problem for the functions $w_{j}(y, t), a_{j}(y, t)$ and $l_{n}(x, t)$. The problem for $b_{j}(y, t)$ given $v_{j}(y, t)$ and $a_{j}(y, t)$ can be separated; the boundary conditions for $b_{j}(y, t)$ are analogous to those for $a_{j}(y, t)$. The functions $d_{j}(y, t)$ can be recovered by quadrature from the second equation (4) up to a function of time. Thus, $w_{j}(y, t), v_{j}(y, t)$, and $a_{j}(y, t)$ are solutions of nonlinear parabolic equations with boundary conditions (5)-(7), (12) and initial data (13) and (14). The last two conditions in (5) and the fourth from (12) are the additional conditions on $f_{j}(t)$.

To simplify (4)-(7), (10)-(14) we introduce the characteristic scales of length and time as well as functions $w_{j}, v_{j}, a_{j}, d_{j}$, and $f_{j}$; namely, the quantities $l_{1}^{0}, l_{1}^{02} / \nu_{1}, \varkappa_{1} a^{0} l_{1}^{0} / \mu_{1}, \varkappa_{2} a^{0} l_{1}^{02} / \mu_{1}, a^{0}, \varkappa_{1} a^{0} l_{1}^{0} / \rho_{1}$, 
and $\varkappa_{1} a^{0} /\left(\rho_{1} l_{1}^{0}\right)$ respectively, where $l_{1}^{0}=$ const $>0$ is the average value of thickness of the first layer of the liquid at $t=0$,

$$
a^{0}=\max _{t \geqslant 0}\left|a_{30}(t)-a_{10}(t)\right|>0 \quad \text { or } \quad a^{0}=\max _{j} \max _{y}\left|a_{j}^{0}(y)\right|>0 .
$$

In the dimensionless variables, some factor appears at the nonlinear terms in (4), the Marangoni number $\mathrm{M}=\varkappa_{1} a^{0} l_{1}^{03} \mu_{1}^{-1} \nu_{1}^{-1}$. The same applies to the kinematic conditions (6); this time for the linear terms containing velocities. Assume that the temperature coefficients of the surface tensions are comparable in their value $\varkappa_{1} \sim \varkappa_{2}$ and $\mathrm{M} \ll 1$. The latter holds either in the thin layers or for large viscosities. Then the nonlinear terms in the equations can be neglected, and the latter become linear. In particular, the kinematic conditions have the form $l_{n t}=0$, i.e., $l_{n}=l_{n}(x)$ for $n=1,2$.

Let us turn to (11). After transition to the dimensionless variables on the right-hand sides, the Weber numbers $\mathrm{We}_{n}=\sigma_{n}^{0} /\left(a^{0} l_{1}^{02} \varkappa_{1}\right)$ appear instead of $\sigma_{1}^{0}$ and $\sigma_{2}^{0}$. In the real conditions, $\mathrm{We}_{n} \gg 1$ for the most of liquid media; for example, for the water-air system We $\sim 10^{6}$. Therefore, for these Weber numbers, (11) assume the form $l_{n x x}=0$; i.e., $l_{n}(x)=\alpha_{n} x+l_{n}^{0}$.

We assume later that $\alpha_{n}=0$ and the separation surfaces are the planes $y=l_{1}^{0}$ and $y=l_{2}^{0}>l_{1}^{0}$, parallel to the solid walls $y=0$ and $y=l_{3}$; in what follows, the index 0 for $l_{n}^{0}$ will be omitted.

\section{A PRIORI ESTIMATES}

Let us present the so-obtained linear problem in its entirety in dimensional form:

$$
\begin{gathered}
w_{j t}=\nu_{j} w_{j y y}+f_{j}(t), \quad j=1,2,3, \\
w_{j}(y, 0)=0, \\
w_{1}(0, t)=0, \quad w_{3}\left(l_{3}, t\right)=0, \\
w_{1}\left(l_{1}, t\right)=w_{2}\left(l_{1}, t\right), \quad w_{2}\left(l_{2}, t\right)=w_{3}\left(l_{2}, t\right), \\
\mu_{2} w_{2 y}\left(l_{1}, t\right)-\mu_{1} w_{1 y}\left(l_{1}, t\right)=-2 \varkappa_{1} a_{1}\left(l_{1}, t\right), \\
\mu_{3} w_{3 y}\left(l_{2}, t\right)-\mu_{2} w_{2 y}\left(l_{2}, t\right)=-2 \varkappa_{2} a_{2}\left(l_{2}, t\right), \\
\int_{0}^{l_{1}} w_{1}(y, t) d y=0, \quad \int_{l_{1}}^{l_{2}} w_{2}(y, t) d y=0, \quad \int_{l_{2}}^{l_{3}} w_{3}(y, t) d y=0,
\end{gathered}
$$

where $0<y<l_{1}$ for $j=1, l_{1}<y<l_{2}$ for $j=2$, and $l_{2}<y<l_{3}$ for $j=3$. Conditions (19) follow from (10) since $\boldsymbol{\tau}_{1}=\boldsymbol{\tau}_{2}=(1,0)$ and $\nabla_{11} \Theta_{1,2} \cdot \boldsymbol{\tau}_{1,2}=2 a_{1,2} x$ according to representation (3) for temperature. The first two equalities in (20) follow from (6), whereas the last is the no-slip condition: $v_{3}\left(l_{3}, t\right)=0$.

Let us write the problem for the functions $a_{j}(y, t)$ :

$$
\begin{gathered}
a_{j t}=\chi_{j} a_{j y y}, \\
a_{j}(y, 0)=a_{j}^{0}(y), \\
a_{1}(0, t)=a_{10}(t), \quad a_{3}\left(l_{3}, t\right)=a_{30}(t), \\
a_{1}\left(l_{1}, t\right)=a_{2}\left(l_{1}, t\right), \quad a_{2}\left(l_{2}, t\right)=a_{3}\left(l_{2}, t\right), \\
k_{1} a_{1 y}\left(l_{1}, t\right)=k_{2} a_{2 y}\left(l_{1}, t\right), \quad k_{2} a_{2 y}\left(l_{2}, t\right)=k_{3} a_{3 y}\left(l_{2}, t\right) .
\end{gathered}
$$

In order to obtain a priori estimates for $w_{j}(y, t)$ and $f_{j}(t)$ of the solution of $(15)-(20)$, it is necessary firstly to infer the estimates for the solutions of initial-boundary value problem (21)-(25). We perform the change of variables:

$$
\begin{gathered}
a_{1}(y, t)=\tilde{a}_{1}(y, t)+\frac{a_{10}(t)}{l_{1}^{2}}\left(y-l_{1}\right)^{2}, \quad a_{2}(y, t)=\tilde{a}_{2}(y, t), \\
a_{3}(y, t)=\tilde{a}_{3}(y, t)+\frac{a_{30}(t)}{\left(l_{3}-l_{2}\right)^{2}}\left(y-l_{2}\right)^{2} .
\end{gathered}
$$


The functions $\tilde{a}_{j}(y, t)$ in their domains satisfy the equations

$$
\begin{array}{r}
\tilde{a}_{1 t}=\chi_{1} \tilde{a}_{1 y y}+\frac{2 \chi_{1} a_{10}(t)}{l_{1}^{2}}-\frac{a_{10}^{\prime}(t)\left(y-l_{1}\right)^{2}}{l_{1}^{2}}, \\
\tilde{a}_{2 t}=\chi_{2} \tilde{a}_{2 y y}, \\
\tilde{a}_{3 t}=\chi_{3} \tilde{a}_{3 y y}+\frac{2 \chi_{3} a_{30}(t)}{\left(l_{3}-l_{2}\right)^{2}}-\frac{a_{30}^{\prime}(t)\left(y-l_{2}\right)^{2}}{\left(l_{3}-l_{2}\right)^{2}},
\end{array}
$$

where the prime denotes differentiation with respect to time.

Boundary conditions (23) for $\tilde{a}_{1}$ and $\tilde{a}_{3}$ become homogeneous, whereas (24) and (25) preserve their form. Initial conditions (22) for $\tilde{a}_{1}$ and $\tilde{a}_{3}$ change:

$$
\begin{gathered}
\tilde{a}_{1}(y, 0)=a_{1}^{0}(y)-\frac{a_{10}(0)}{l_{1}^{2}}\left(y-l_{1}\right)^{2} \equiv \tilde{a}_{1}^{0}(y), \\
\tilde{a}_{3}(y, 0)=a_{3}^{0}(y)-\frac{a_{30}(0)}{\left(l_{3}-l_{2}\right)^{2}}\left(y-l_{2}\right)^{2} \equiv \tilde{a}_{3}^{0}(y) .
\end{gathered}
$$

Introduce the notations:

$$
g_{1}(y, t)=\frac{2 \chi_{1} a_{10}(t)}{l_{1}^{2}}-\frac{a_{10}^{\prime}(t)\left(y-l_{1}\right)^{2}}{l_{1}^{2}}, \quad g_{3}(y, t)=\frac{2 \chi_{3} a_{30}(t)}{\left(l_{3}-l_{2}\right)^{2}}-\frac{a_{30}^{\prime}(t)\left(y-l_{2}\right)^{2}}{\left(l_{3}-l_{2}\right)^{2}} .
$$

Let us multiply (27)-(29) by $\rho_{1} c_{1} \tilde{a}_{1}, \rho_{2} c_{2} \tilde{a}_{2}$, and $\rho_{3} c_{3} \tilde{a}_{3}$ and integrate over the segments $\left[0, l_{1}\right],\left[l_{1}, l_{2}\right]$, and $\left[l_{2}, l_{3}\right]$ taking into account (24) and (25). Then add up the results. We infer that

$$
\begin{gathered}
\frac{d A(t)}{d t}+k_{1} \int_{0}^{l_{1}} \tilde{a}_{1 y}^{2} d y+k_{2} \int_{l_{1}}^{l_{2}} \tilde{a}_{2 y}^{2} d y+k_{3} \int_{l_{2}}^{l_{3}} \tilde{a}_{3 y}^{2} d y \\
=\rho_{1} c_{1} \int_{0}^{l_{1}} g_{1}(y, t) \tilde{a}_{1}(y, t) d y+\rho_{3} c_{3} \int_{l_{2}}^{l_{3}} g_{3}(y, t) \tilde{a}_{3}(y, t) d y, \\
A(t)=\frac{1}{2} \rho_{1} c_{1} \int_{0}^{l_{1}} \tilde{a}_{1}^{2} d y+\frac{1}{2} \rho_{2} c_{2} \int_{l_{1}}^{l_{2}} \tilde{a}_{2}^{2} d y+\frac{1}{2} \rho_{3} c_{3} \int_{l_{2}}^{l_{3}} \tilde{a}_{3}^{2} d y,
\end{gathered}
$$

where $c_{j}=k_{j} /\left(\rho_{j} \chi_{j}\right)$ are the coefficients of the specific heat capacity. Since on the solid walls $\tilde{a}_{1}(0, t)=0$ and $\tilde{a}_{3}\left(l_{3}, t\right)=0$, while for $\tilde{a}_{1}, \tilde{a}_{2}$, and $\tilde{a}_{3}$ the conditions (24) and (25) are fulfilled; therefore,

$$
\int_{0}^{l_{1}} \tilde{a}_{1}^{2} d y+\int_{l_{1}}^{l_{2}} \tilde{a}_{2}^{2} d y+\int_{l_{2}}^{l_{3}} \tilde{a}_{3}^{2} d y \leq M_{1}\left(k_{1} \int_{0}^{l_{1}} \tilde{a}_{1 y}^{2} d y+k_{2} \int_{l_{1}}^{l_{2}} \tilde{a}_{2 y}^{2} d y+k_{3} \int_{l_{2}}^{l_{3}} \tilde{a}_{3 y}^{2} d y\right)
$$

with a positive minimum constant $M_{1}<\infty$ [15]. Hence, the left-hand side in (32) is at least

$$
\frac{d A(t)}{d t}+2 \delta A(t), \quad \delta=\frac{1}{M_{1}} \min _{j}\left(\frac{\chi_{j}}{k_{j}}\right) .
$$

As for the right-hand side, it does not exceed

$$
\begin{gathered}
\rho_{1} c_{1}\left(\int_{0}^{l_{1}} g_{1}^{2} d y\right)^{1 / 2}\left(\int_{0}^{l_{1}} \tilde{a}_{1}^{2} d y\right)^{1 / 2}+\rho_{3} c_{3}\left(\int_{l_{2}}^{l_{3}} g_{3}^{2} d y\right)^{1 / 2}\left(\int_{l_{2}}^{l_{3}} \tilde{a}_{3}^{2} d y\right)^{1 / 2} \\
\leq\left[\left(2 \rho_{1} c_{1} \int_{0}^{l_{1}} g_{1}^{2} d y\right)^{1 / 2}+\left(2 \rho_{3} c_{3} \int_{l_{2}}^{l_{3}} g_{3}^{2} d y\right)^{1 / 2}\right] \sqrt{A(t)} \equiv G(t) \sqrt{A(t)}
\end{gathered}
$$


Thus, from (32) with (34) and (35) we derive $\frac{d A(t)}{d t}+2 \delta A(t) \leq G(t) \sqrt{A(t)}$, and so

$$
A(t) \leq\left[\sqrt{A(0)}+\frac{1}{2} \int_{0}^{t} e^{\delta \tau} G(\tau) d \tau\right]^{2} e^{-2 \delta t} .
$$

According to (33),

$$
A(0)=\frac{1}{2} \rho_{1} c_{1} \int_{0}^{l_{1}}\left(\tilde{a}_{1}^{0}(y)\right)^{2} d y+\frac{1}{2} \rho_{2} c_{2} \int_{l_{1}}^{l_{2}}\left(a_{2}^{0}(y)\right)^{2} d y+\frac{1}{2} \rho_{3} c_{3} \int_{l_{2}}^{l_{3}}\left(\tilde{a}_{3}^{0}(y)\right)^{2} d y
$$

where $\tilde{a}_{1}^{0}(y)$ and $\tilde{a}_{3}^{0}(y)$ are given by (30). Then, using (31), we obtain

$$
\begin{gathered}
\int_{0}^{l_{1}} g_{1}^{2}(y, t) d y \leq \frac{4 \chi_{1}^{2} a_{10}^{2}(t)}{l_{1}^{3}}+\frac{1}{5} l_{1}\left(a_{10}^{\prime}(t)\right)^{2}, \\
\int_{l_{2}}^{l_{3}} g_{3}^{2}(y, t) d y \leq \frac{4 \chi_{3}^{2} a_{30}^{2}(t)}{\left(l_{3}-l_{2}\right)^{3}}+\frac{1}{5}\left(l_{3}-l_{2}\right)\left(a_{30}^{\prime}(t)\right)^{2} .
\end{gathered}
$$

By the definition of $G(t)$ (see (35)), we have

$$
\begin{aligned}
G(t) \leq 2 \sqrt{\rho_{1} c_{1}}\left(\frac{2 \chi_{1}\left|a_{10}(t)\right|}{l_{1}^{3 / 2}+}+\frac{1}{\sqrt{5}} \sqrt{l_{1}}\left|a_{10}^{\prime}(t)\right|\right) \\
+2 \sqrt{\rho_{3} c_{3}}\left(\frac{2 \chi_{3}\left|a_{30}(t)\right|}{\left(l_{3}-l_{2}\right)^{3 / 2}}+\frac{1}{\sqrt{5}} \sqrt{\left(l_{3}-l_{2}\right)}\left|a_{30}^{\prime}(t)\right|\right)
\end{aligned}
$$

because of $\sqrt{x^{2}+y^{2}} \leq|x|+|y|$ for all $x, y \in R^{1}$.

Remark 1. If the integrals

$$
\int_{0}^{\infty} e^{\delta \tau}\left|a_{k 0}(\tau)\right| d \tau, \quad \int_{0}^{\infty} e^{\delta \tau}\left|a_{k 0}^{\prime}(\tau)\right| d \tau, \quad k=1,3,
$$

converge then, by (36)-(39), $A(t)$ tends to zero with time by an exponential law. Physically it means that the thermal effects are rather small.

In view of (38) for $G(t)$, we see that (36) assumes the form

$$
\begin{aligned}
& A(t) \leq\left[\sqrt{A(0)}+\sqrt{\rho_{1} c_{1}}\left(\frac{2 \chi_{1}}{l_{1}^{3 / 2}} \int_{0}^{t} e^{\delta \tau}\left|a_{10}(\tau)\right| d \tau+\sqrt{\frac{l_{1}}{5}} \int_{0}^{t} e^{\delta \tau}\left|a_{10}^{\prime}(\tau)\right| d \tau\right)\right. \\
& \left.+\sqrt{\rho_{3} c_{3}}\left(\frac{2 \chi_{3}}{\left(l_{3}-l_{2}\right)^{3 / 2}} \int_{0}^{t} e^{\delta \tau}\left|a_{30}(\tau)\right| d \tau+\sqrt{\frac{l_{3}-l_{2}}{5}} \int_{0}^{t} e^{\delta \tau}\left|a_{30}^{\prime}(\tau)\right| d \tau\right)\right]^{2} e^{-2 \delta t} .
\end{aligned}
$$

Let us rewrite (27)-(29) as $\tilde{a}_{j t}-\chi_{j} \tilde{a}_{j y y}=g_{j}(y, t), g_{2}=0$, square each equation, and multiply by $\rho_{j} c_{j}$. Then let us integrate with respect to time and $y$ in the respective domains and sum up the results. Using 
the boundary and initial conditions, we arrive at the integral equality

$$
\begin{gathered}
\int_{0}^{t}\left[\rho_{1} c_{1} \int_{0}^{l_{1}}\left(\tilde{a}_{1 t}^{2}+\chi_{1}^{2} \tilde{a}_{1 y y}^{2}\right) d y+\rho_{2} c_{2} \int_{l_{1}}^{l_{2}}\left(\tilde{a}_{2 t}^{2}+\chi_{2}^{2} \tilde{a}_{2 y y}^{2}\right) d y+\rho_{3} c_{3} \int_{l_{2}}^{l_{3}}\left(\tilde{a}_{3 t}^{2}+\chi_{3}^{2} \tilde{a}_{3 y y}^{2}\right) d y\right] d t \\
+k_{1} \int_{0}^{l_{1}} \tilde{a}_{1 y}^{2}(y, t) d y+k_{2} \int_{l_{1}}^{l_{2}} \tilde{a}_{2 y}^{2}(y, t) d y+k_{3} \int_{l_{2}}^{l_{3}} \tilde{a}_{3 y}^{2}(y, t) d y \\
=k_{1} \int_{0}^{l_{1}} \tilde{a}_{10 y}^{2}(y) d y+k_{2} \int_{l_{1}}^{l_{2}} \tilde{a}_{20 y}^{2}(y) d y+k_{3} \int_{l_{2}}^{l_{3}} \tilde{a}_{30 y}^{2}(y) d y \\
+\rho_{1} c_{1} \int_{0}^{t} \int_{0}^{l_{1}} g_{1}^{2}(y, t) d y d t+\rho_{3} c_{3} \int_{0}^{t} \int_{l_{2}}^{l_{3}} g_{3}^{2}(y, t) d y d t .
\end{gathered}
$$

Considering (37), we obtain from (41) the estimates

$$
\int_{0}^{l_{1}} \tilde{a}_{1 y}^{2}(y, t) d y \leq \frac{F(t)}{k_{1}}, \quad \int_{l_{1}}^{l_{2}} \tilde{a}_{2 y}^{2}(y, t) d y \leq \frac{F(t)}{k_{2}}, \quad \int_{l_{2}}^{l_{3}} \tilde{a}_{3 y}^{2}(y, t) d y \leq \frac{F(t)}{k_{3}}
$$

with the function

$$
\begin{aligned}
F(t)=k_{1} \int_{0}^{l_{1}}\left(\tilde{a}_{1 y}^{0}(y)\right)^{2} d y & +k_{2} \int_{l_{1}}^{l_{2}}\left(a_{2 y}^{0}(y)\right)^{2} d y+k_{3} \int_{l_{2}}^{l_{3}}\left(\tilde{a}_{3 y}^{0}(y)\right)^{2} d y \\
& +2 \rho_{1} c_{1}\left[\frac{4 \chi_{1}^{2}}{l_{1}^{3}} \int_{0}^{t} a_{10}^{2}(\tau) d \tau+\frac{1}{5} l_{1} \int_{0}^{t}\left(a_{10}^{\prime}(\tau)\right)^{2} d \tau\right] \\
& +2 \rho_{3} c_{3}\left[\frac{4 \chi_{3}^{2}}{\left(l_{3}-l_{2}\right)} \int_{0}^{t} a_{30}^{2}(\tau) d \tau+\frac{1}{5}\left(l_{3}-l_{2}\right)^{2} \int_{0}^{t}\left(a_{30}^{\prime}(\tau)\right)^{2} d \tau\right] .
\end{aligned}
$$

Remark 2. The convergence of (39) also yields the convergence as $t \rightarrow \infty$ of the integrals in the expression of $F(t)$ in (43).

Returning to (26), from (36), (38), (42), (43), and the Cauchy-Bunyakovskii Inequality we obtain the uniform estimates in $y$ :

$$
\begin{gathered}
\left|a_{1}(y, t)\right| \leqslant\left(\frac{8 A(t) F(t)}{\rho_{1} c_{1} k_{1}}\right)^{1 / 4}+h_{1}(t) e^{-\delta t} \\
\left|a_{3}(y, t)\right| \leq\left(\frac{8 A(t) F(t)}{\rho_{3} c_{3} k_{3}}\right)^{1 / 4}+h_{3}(t) e^{-\delta t} \\
\left|a_{2}(y, t)\right| \leq(8 A(t) F(t))^{1 / 4}\left(\left(\rho_{2} c_{2} k_{2}\right)^{-1 / 2}+\left(\rho_{1} c_{1} k_{1}\right)^{-1 / 2}\right)^{1 / 2} .
\end{gathered}
$$

Remark 3. Since the integrals (39) converge, we have $a_{j 0}(\tau) \leq h_{j}(\tau) e^{-\delta \tau}$ and $a_{j 0}^{\prime}(\tau) \leq h_{j}^{1}(\tau) e^{-\delta \tau}$, whereas

$$
\int_{0}^{\infty}\left|h_{j}(\tau)\right| d \tau<\infty, \quad \int_{0}^{\infty}\left|h_{j}^{1}(\tau)\right| d \tau<\infty
$$


The following holds:

Theorem 1. If the integrals (39) converge then the solution of (21)-(25) tends to zero as $t \rightarrow \infty$. Moreover, we have the estimates (44)-(46) for the convergence rate.

Indeed, according to (43) the function $F(t)$ is bounded by a constant, and from (40) $A(t) \sim e^{-2 \delta t}$; therefore, (44)-(46) prove the theorem.

Let us return to (15)-(20). Note the following identity

$$
\begin{aligned}
\frac{d E(t)}{d t}+\mu_{1} \int_{0}^{l_{1}} w_{1 y}^{2} d y+\mu_{2} \int_{l_{1}}^{l_{2}} w_{2 y}^{2} d y & +\mu_{3} \int_{l_{2}}^{l_{3}} w_{3 y}^{2} d y \\
& =2 \varkappa_{1} a_{1}\left(l_{1}, t\right) w_{1}\left(l_{1}, t\right)+2 \varkappa_{2} a_{3}\left(l_{2}, t\right) w_{3}\left(l_{2}, t\right), \\
E(t) & =\frac{1}{2} \rho_{1} \int_{0}^{l_{1}} w_{1}^{2} d y+\frac{1}{2} \rho_{2} \int_{l_{1}}^{l_{2}} w_{2}^{2} d y+\frac{1}{2} \rho_{3} \int_{l_{2}}^{l_{3}} w_{3}^{2} d y .
\end{aligned}
$$

Observe that, using (26), we have $a_{1}\left(l_{1}, t\right)=\tilde{a}_{1}\left(l_{1}, t\right)$ and $a_{3}\left(l_{2}, t\right)=\tilde{a}_{3}\left(l_{2}, t\right)$ in (47). Now,

$$
\left|w_{1}\left(l_{1}, t\right)\right| \leq \sqrt{l_{1}}\left(\int_{0}^{l_{1}} w_{1 y}^{2} d y\right)^{1 / 2}, \quad\left|w_{3}\left(l_{2}, t\right)\right| \leq \sqrt{l_{3}-l_{2}}\left(\int_{l_{2}}^{l_{3}} w_{3 y}^{2} d y\right)^{1 / 2}
$$

and so the right-hand side of (47) is estimated from above as follows: for all $\varepsilon_{1}>0$ and $\varepsilon_{2}>0$, we have

$$
\begin{aligned}
\mid 2 \varkappa_{1} \tilde{a}_{1}\left(l_{1}, t\right) w_{1}\left(l_{1}, t\right)+ & 2 \varkappa_{2} \tilde{a}_{3}\left(l_{2}, t\right) w_{3}\left(l_{2}, t\right) \mid \\
& \leq \frac{\varkappa_{1}}{\varepsilon_{1}} \tilde{a}_{1}^{2}\left(l_{1}, t\right)+\frac{\varkappa_{2}}{\varepsilon_{2}} \tilde{a}_{3}^{2}\left(l_{2}, t\right)+\varkappa_{1} l_{1} \varepsilon_{1} \int_{0}^{l_{1}} w_{1 y}^{2} d y+\varkappa_{2}\left(l_{3}-l_{2}\right) \varepsilon_{2} \int_{l_{2}}^{l_{3}} w_{3 y}^{2} d y .
\end{aligned}
$$

Choosing $\varepsilon_{1}<\mu_{1} / \varkappa_{1} l_{1}$ and $\varepsilon_{2}<\mu_{2} / \varkappa_{2}\left(l_{3}-l_{2}\right)$ and applying (44) and (45), we find from (47) that

$$
\begin{aligned}
\frac{d E}{d t}+\left(\mu_{1}-\varkappa_{1} l_{1} \varepsilon_{1}\right) \int_{0}^{l_{1}} w_{1 y}^{2} d y+\mu_{2} \int_{l_{1}}^{l_{2}} w_{2 y}^{2} d y & +\left[\mu_{3}-\varkappa_{2}\left(l_{3}-l_{2}\right) \varepsilon_{2}\right] \int_{l_{2}}^{l_{3}} w_{3 y}^{2} d y \\
& \leq 4\left(\frac{\varkappa_{1}}{\varepsilon_{1}} \frac{1}{\sqrt{\rho_{1} c_{1} k_{1}}}+\frac{\varkappa_{2}}{\varepsilon_{2}} \frac{1}{\sqrt{\rho_{3} c_{3} k_{3}}}\right) \sqrt{A(t) F(t)} .
\end{aligned}
$$

Owing to

$$
\int_{0}^{l_{1}} w_{1}^{2} d y+\int_{l_{1}}^{l_{2}} w_{2}^{2} d y+\int_{l_{2}}^{l_{3}} w_{3}^{2} d y \leq \frac{l_{3}^{2}}{2}\left(\int_{0}^{l_{1}} w_{1 y}^{2} d y+\int_{l_{1}}^{l_{2}} w_{2 y}^{2} d y+\int_{l_{2}}^{l_{3}} w_{3 y}^{2} d y\right),
$$

we can rewrite $(48)$ as

$$
\frac{d E}{d t}+4 \delta_{1} E \leq H(t), \quad \delta_{1}=\frac{1}{l_{3}^{2}} \min \left\{\frac{\mu_{1}-\varkappa_{1} l_{1} \varepsilon_{1}}{\rho_{1}}, \frac{\mu_{2}}{\rho_{2}}, \frac{\mu_{3}-\varkappa_{2}\left(l_{3}-l_{2}\right) \varepsilon_{2}}{\rho_{3}}\right\},
$$

where

$$
H(t)=4\left(\frac{\varkappa_{1}}{\varepsilon_{1}} \frac{1}{\sqrt{\rho_{1} c_{1} k_{1}}}+\frac{\varkappa_{2}}{\varepsilon_{2}} \frac{1}{\sqrt{\rho_{3} c_{3} k_{3}}}\right) \sqrt{A(t) F(t)}
$$


By (40), $H(t)$ can be estimated from above as follows:

$$
\begin{aligned}
H(t) \leq 4\left(\frac{\varkappa_{1}}{\varepsilon_{1}}\right. & \left.\frac{1}{\sqrt{\rho_{1} c_{1} k_{1}}}+\frac{\varkappa_{2}}{\varepsilon_{2}} \frac{1}{\sqrt{\rho_{3} c_{3} k_{3}}}\right) \sqrt{F(t)} \\
& \times\left[\sqrt{A(0)}+\sqrt{\rho_{1} c_{1}}\left(\frac{2 \chi_{1}}{l_{1}^{3 / 2}} \int_{0}^{t} e^{\delta \tau}\left|a_{10}(\tau)\right| d \tau+\sqrt{\frac{l_{1}}{5}} \int_{0}^{t} e^{\delta \tau}\left|a_{10}^{\prime}(\tau)\right| d \tau\right)\right. \\
& \left.+\sqrt{\rho_{3} c_{3}}\left(\frac{2 \chi_{3}}{\left(l_{3}-l_{2}\right)^{3 / 2}} \int_{0}^{t} e^{\delta \tau}\left|a_{30}(\tau)\right| d \tau+\sqrt{\frac{l_{3}-l_{2}}{5}} \int_{0}^{t} e^{\delta \tau}\left|a_{30}^{\prime}(\tau)\right| d \tau\right)\right] e^{-\delta t} .
\end{aligned}
$$

The function $F(t)$ in (51) is given by (43) and bounded by a constant (see Remark 2).

From (49) we obtain

$$
E(t) \leq e^{-4 \delta_{1} t} \int_{0}^{t} e^{4 \delta_{1} \tau} H(\tau) d \tau
$$

Hence, we find the estimates of the $L_{2}$-norms of $w_{j}(y, t)$ on their respective domains

$$
\int_{0}^{l_{1}} w_{1}^{2} d y \leq \frac{2}{\rho_{1}} E(t), \quad \int_{l_{1}}^{l_{2}} w_{2}^{2} d y \leq \frac{2}{\rho_{2}} E(t), \quad \int_{l_{2}}^{l_{3}} w_{3}^{2} d y \leq \frac{2}{\rho_{3}} E(t) .
$$

To obtain some analogous estimates for the derivatives $w_{j y}$, we carry out the substitution

$$
\begin{gathered}
w_{1}(y, t)=\widetilde{w}_{1}(y, t)+\frac{2 \varkappa_{1} a_{1}\left(l_{1}, t\right)}{\mu_{1} l_{1}^{2}}\left(2 y^{3}-3 l_{1} y^{2}+l_{1}^{2} y\right), \quad w_{2}(y, t)=\widetilde{w}_{2}(y, t), \\
w_{3}(y, t)=\widetilde{w}_{3}(y, t)-\frac{2 \varkappa_{2} a_{2}\left(l_{2}, t\right)}{\mu_{3}\left(l_{3}-l_{2}\right)^{2}}\left[2 y^{3}-3\left(l_{2}+l_{3}\right) y^{2}+\left(l_{2}^{2}+l_{3}^{2}+4 l_{2} l_{3}\right) y-l_{2} l_{3}\left(l_{2}+l_{3}\right)\right] .
\end{gathered}
$$

In this case, the boundary conditions $(19)$ for $\widetilde{w}_{j}(y, t)$ become homogeneous, whereas (17), (18), and (20) have the same form. In (15) for $j=1$ and $j=3$, the right-hand sides change:

$$
\begin{array}{r}
\tilde{f}_{1}(y, t)=f_{1}(t)-\frac{2 \varkappa_{1} a_{1 t}\left(l_{1}, t\right)}{\mu_{1} l_{1}^{2}}\left(2 y^{3}-3 l_{1} y^{2}+l_{1}^{2} y\right)+\frac{12 \varkappa_{1} \nu_{1} a_{1}\left(l_{1}, t\right)}{\mu_{1} l_{1}^{2}}\left(2 y-l_{1}\right) \\
\equiv f_{1}(t)+Q_{1}(y, t), \\
\tilde{f}_{3}(y, t)=f_{3}(t)+\frac{2 \varkappa_{2} a_{2 t}\left(l_{2}, t\right)}{\mu_{3}\left(l_{3}-l_{2}\right)^{2}}\left[2 y^{3}-3\left(l_{2}+l_{3}\right) y^{2}+\left(l_{2}^{2}+l_{3}^{2}+4 l_{2} l_{3}\right) y-l_{2} l_{3}\left(l_{2}+l_{3}\right)\right] \\
-\frac{12 \varkappa_{2} \nu_{3} a_{2}\left(l_{2}, t\right)}{\mu_{3}\left(l_{3}-l_{2}\right)^{2}}\left[2 y-\left(l_{2}+l_{3}\right)\right] \equiv f_{3}(t)+Q_{3}(t) .
\end{array}
$$

Initial data (16) are inhomogeneous for $j=1$ and $j=3$ :

$$
\begin{gathered}
\widetilde{w}_{1}(y, 0)=-\frac{2 \varkappa_{1} a_{1}\left(l_{1}, 0\right)}{\mu_{1} l_{1}^{2}}\left(2 y^{3}-3 l_{1} y^{2}+l_{1}^{2} y\right) \equiv \widetilde{w}_{10}(y) \\
\widetilde{w}_{3}(y, 0)=\frac{2 \varkappa_{2} a_{2}\left(l_{2}, 0\right)}{\mu_{3}\left(l_{3}-l_{2}\right)^{2}}\left[2 y^{3}-3\left(l_{2}+l_{3}\right) y^{2}+\left(l_{2}^{2}+l_{3}^{2}+4 l_{2} l_{3}\right) y-l_{2} l_{3}\left(l_{2}+l_{3}\right)\right] \equiv \widetilde{w}_{30}(y) .
\end{gathered}
$$


Multiply the equations for $\widetilde{w}_{j}(y, t)$ by $\rho_{j} \widetilde{w}_{j t}$ and sum up the results:

$$
\begin{aligned}
& \rho_{1} \int_{0}^{l_{1}} \widetilde{w}_{1 t}^{2} d y+\rho_{2} \int_{l_{1}}^{l_{2}} \widetilde{w}_{2 t}^{2} d y+\rho_{3} \int_{l_{2}}^{l_{3}} \widetilde{w}_{3 t}^{2} d y \\
&+\frac{\partial}{\partial t}\left[\frac{1}{2} \mu_{1} \int_{0}^{l_{1}} \widetilde{w}_{1 y}^{2} d y+\frac{1}{2} \mu_{2} \int_{l_{1}}^{l_{2}} \widetilde{w}_{2 y}^{2} d y+\frac{1}{2} \mu_{3} \int_{l_{2}}^{l_{3}} \widetilde{w}_{3 y}^{2} d y\right] \\
& \quad=\rho_{1} \int_{0}^{l_{1}} Q_{1}(y, t) \widetilde{w}_{1 t} d y+\rho_{3} \int_{l_{2}}^{l_{3}} Q_{3}(y, t) \widetilde{w}_{3 t} d y .
\end{aligned}
$$

From (57) we derive the estimate

$$
\begin{aligned}
\frac{1}{2} \mu_{1} \int_{0}^{l_{1}} \widetilde{w}_{1 y}^{2} d y+\frac{1}{2} \mu_{2} \int_{l_{1}}^{l_{2}} \widetilde{w}_{2 y}^{2} d y+\frac{1}{2} \mu_{3} \int_{l_{2}}^{l_{3}} \widetilde{w}_{3 y}^{2} d y & \leq \frac{1}{2} \mu_{1} \int_{0}^{l_{1}} \widetilde{w}_{10 y}^{2} d y+\frac{1}{2} \mu_{3} \int_{l_{2}}^{l_{3}} \widetilde{w}_{30 y}^{2} d y \\
& +\frac{1}{2}\left[\frac{\rho_{1}}{\varepsilon_{3}} \int_{0}^{t} \int_{0}^{l_{1}} Q_{1}^{2}(y, \tau) d y d \tau+\frac{\rho_{3}}{\varepsilon_{4}} \int_{0}^{t} \int_{l_{2}}^{l_{3}} Q_{3}^{2}(y, \tau) d y d \tau\right] .
\end{aligned}
$$

By the definitions of $Q_{1}(y, t)$ and $Q_{2}(y, t)$, from (55), (56), and (58) we obtain the boundedness of the quantities

$$
\int_{0}^{l_{1}} \widetilde{w}_{1 y}^{2} d y, \quad \int_{l_{1}}^{l_{2}} \widetilde{w}_{2 y}^{2} d y, \quad \int_{l_{2}}^{l_{3}} \widetilde{w}_{3 y}^{2} d y
$$

provided that

$$
\int_{0}^{\infty} a_{1}^{2}\left(l_{1}, \tau\right) d \tau, \quad \int_{0}^{\infty} a_{1 \tau}^{2}\left(l_{1}, \tau\right) d \tau, \quad \int_{0}^{\infty} a_{2}^{2}\left(l_{2}, \tau\right) d \tau, \quad \int_{0}^{\infty} a_{2 \tau}^{2}\left(l_{2}, \tau\right) d \tau
$$

converge. The convergence $\int_{0}^{\infty} a_{1}^{2}\left(l_{1}, \tau\right) d \tau$ and $\int_{0}^{\infty} a_{2}^{2}\left(l_{2}, \tau\right) d \tau$ follows from (44) and (46). In order to demonstrate the boundedness of

$$
\int_{0}^{\infty} a_{1 \tau}^{2}\left(l_{1}, \tau\right) d \tau, \quad \int_{0}^{\infty} a_{2 \tau}^{2}\left(l_{2}, \tau\right) d \tau
$$

we have to estimate $a_{j t}(y, t)$. To this end, it suffices to differentiate (21) and (23)-(25) with respect to $t$. In result, the problem arises for $a_{j t}(y, t)$ that is analogous to (21)-(25) after replacement of $(22)$ by

$$
a_{j t}(y, 0)=\psi_{j y y} a^{0}(y) .
$$

If, in addition to the convergence of (39), we require the convergence of the integrals

$$
\int_{0}^{\infty} e^{\delta \tau}\left|a_{j 0}^{\prime \prime}(\tau)\right| d \tau, \quad j=1,3
$$


then, using Remark 3, we estimate

$$
\begin{gathered}
\left|a_{1 t}(y, t)\right| \leq\left(\frac{8 A_{1}(t) F_{1}(t)}{\rho_{1} c_{1} k_{1}}\right)^{1 / 4}+h_{1}^{1}(t) e^{-\delta t}, \\
\left|a_{3 t}(y, t)\right| \leq\left(\frac{8 A_{1}(t) F_{1}(t)}{\rho_{3} c_{3} k_{3}}\right)^{1 / 4}+h_{3}^{1}(t) e^{-\delta t}, \\
\left|a_{2 t}(y, t)\right| \leqslant\left(8 A_{1}(t) F_{1}(t)\right)^{1 / 4}\left(\left(\rho_{2} c_{2} k_{2}\right)^{-1 / 2}+\left(\rho_{1} c_{1} k_{1}\right)^{-1 / 2}\right)^{1 / 2} .
\end{gathered}
$$

In (61), $A_{1}(t) \sim e^{-2 \delta t}$ and $F_{1}(t)$ is bounded by some constant.

According to (54) and (59), the following integrals are bounded for all $t>0$ :

$$
\int_{0}^{l_{1}} w_{1 y}^{2} d y, \quad \int_{l_{1}}^{l_{2}} w_{2 y}^{2} d y, \quad \int_{l_{2}}^{l_{3}} w_{3 y}^{2} d y .
$$

Using these facts together with (53), we find the estimates

$$
\left|w_{1}(y, t)\right| \leq C_{1}(E(t))^{1 / 4}, \quad\left|w_{2}(y, t)\right| \leq C_{2}(E(t))^{1 / 4}, \quad\left|w_{3}(y, t)\right| \leq C_{3}(E(t))^{1 / 4},
$$

uniform on their respective domains of definition, and $C_{j}>0$ are constants.

Theorem 2. Let the integrals (39) and (60) converge. Then the solution of (15)-(20) converges to zero as $t \rightarrow \infty$, whereas (62) are valid for the convergence rate, where $E(t)$ satisfies (52).

Theorem 2 and Remark 3 demonstrate that, under these conditions, the deceleration of the liquids occurs with time due to friction on walls.

Now, let us proceed to estimating $f_{j}(t)$. To that end, we multiply (15) by $y\left(l_{1}-y\right)$ for $j=1$, by $\left(y-l_{1}\right)\left(l_{2}-y\right)$ for $j=2$, and by $\left(l_{3}-y\right)\left(y-l_{2}\right)$ for $j=3$; and then we integrate over the respective domains:

$$
\begin{aligned}
\frac{1}{6} l_{1}^{3} f_{1}(t) & =\nu_{1} \int_{0}^{l_{1}}\left(l_{1}-2 y\right) w_{1 y} d y+\int_{0}^{l_{1}} y\left(l_{1}-y\right) w_{1 t} d y, \\
\frac{1}{6}\left(l_{2}-l_{1}\right)^{3} f_{2}(t) & =\nu_{2} \int_{l_{1}}^{l_{2}}\left(l_{1}+l_{2}-2 y\right) w_{2 y} d y+\int_{l_{1}}^{l_{2}}\left(y-l_{1}\right)\left(l_{2}-y\right) w_{2 t} d y, \\
\frac{1}{6}\left(l_{3}-l_{2}\right)^{3} f_{3}(t) & =\nu_{3} \int_{l_{2}}^{l_{3}}\left(l_{2}+l_{3}-2 y\right) w_{3 y} d y+\int_{l_{2}}^{l_{3}}\left(y-l_{2}\right)\left(l_{3}-y\right) w_{3 t} d y .
\end{aligned}
$$

In order to obtain the boundedness of $\left|f_{j}(t)\right|$, we estimate $\int_{\Omega_{j}} w_{j t}^{2} d y$. Make the substitution

$$
w_{j t}(y, t)=\varphi(y, t), \quad f_{j t}(t)=\tilde{f}_{j}(t) .
$$

Now (15) can be rewritten as $\varphi_{j t}(y, t)=\nu_{j} \varphi_{j y y}(y, t)+\tilde{f}(t)$. Initial and boundary conditions (16)-(18) and (20) preserve their form, whereas (19) changes:

$$
\mu_{2} \varphi_{2 y}\left(l_{1}, t\right)-\mu_{1} \varphi_{1 y}\left(l_{1}, t\right)=-2 \varkappa_{1} a_{1 t}\left(l_{1}, t\right), \quad \mu_{3} \varphi_{3 y}\left(l_{2}, t\right)-\mu_{2} \varphi_{2 y}\left(l_{2}, t\right)=-2 \varkappa_{2} a_{2 t}\left(l_{2}, t\right) .
$$

For the functions $\varphi(y, t)$ we obtain some problem that is analogous to (15)-(20). Therefore, using (64) and (61), we have

$$
\int_{0}^{l_{1}} w_{1 t}^{2} d y \leq \frac{2}{\rho_{1}} E_{1}(t), \quad \int_{l_{1}}^{l_{2}} w_{2 t}^{2} d y \leq \frac{2}{\rho_{2}} E_{1}(t), \quad \int_{l_{2}}^{l_{3}} w_{3 t}^{2} d y \leq \frac{2}{\rho_{3}} E_{1}(t) .
$$


The function $E_{1}(t)$ in (65) can be estimated from above as follows:

$$
\begin{gathered}
E_{1}(t) \leq e^{-4 \delta_{1} t} \int_{0}^{t} e^{4 \delta_{1} \tau} H_{1}(\tau) d \tau \\
H_{1}(t) \leq 4\left(\frac{\varkappa_{1}}{\varepsilon_{1}} \frac{1}{\sqrt{\rho_{1} c_{1} k_{1}}}+\frac{\varkappa_{2}}{\varepsilon_{2}} \frac{1}{\sqrt{\rho_{3} c_{3} k_{3}}}\right) \sqrt{F_{1}(t) A_{1}(t)} .
\end{gathered}
$$

Hence, using the Cauchy-Bunyakovskii Inequality together with (54), (58), and (65), it is easy to obtain from (63) the boundedness of $\left|f_{j}(t)\right|$ for all $t \geq 0$ if (39) and (60) converge. More precise estimates can be obtained if we use the equalities

$$
\begin{gathered}
\int_{0}^{l_{1}} w_{1 y} d y=w_{1}\left(l_{1}, t\right), \quad \int_{l_{1}}^{l_{2}} w_{2 y} d y=w_{2}\left(l_{2}, t\right)-w_{2}\left(l_{1}, t\right), \quad \int_{l_{2}}^{l_{3}} w_{3 y} d y=-w_{3}\left(l_{2}, t\right), \\
\int_{0}^{l_{1}} y w_{1 y} d y=l_{1} w_{1}\left(l_{1}, t\right), \quad \int_{l_{1}}^{l_{2}} y w_{2 y} d y=l_{2} w_{2}\left(l_{2}, t\right)-l_{1} w_{2}\left(l_{1}, t\right), \\
\int_{l_{2}}^{l_{3}} y w_{3 y} d y=-l_{2} w_{3}\left(l_{2}, t\right) .
\end{gathered}
$$

The first three equalities in (67) are obvious, whereas the others are obtained by integrating (20) by parts.

Using (67) and (18), we can reduce the first terms on the right-hand sides of (65) to the form

$$
-\nu_{1} l_{1} w_{1}\left(l_{1}, t\right), \quad-\nu_{2}\left(l_{2}-l_{1}\right)\left(w_{1}\left(l_{1}, t\right)+w_{3}\left(l_{2}, t\right)\right), \quad-\nu_{3}\left(l_{3}-l_{2}\right) w_{3}\left(l_{2}, t\right) .
$$

Hence, if (39) and (60) converge then, using (68) and the Cauchy-Bunyakovskii Inequality together with (52), (62), (65), and (66), we infer

$$
\begin{gathered}
\left|f_{1}(t)\right| \leqslant\left(\frac{12}{5 l_{1} \rho_{1}} \int_{0}^{t} e^{4 \delta_{1} \tau} H_{1}(\tau) d \tau\right)^{1 / 2} e^{-2 \delta_{1} t}-\frac{6 \nu_{1} C_{1}}{l_{1}^{2}}\left(\int_{0}^{t} e^{4 \delta_{1} \tau} H(\tau) d \tau\right)^{1 / 4} e^{-\delta_{1} t}, \\
\left|f_{2}(t)\right| \leq\left(\frac{12}{5\left(l_{2}-l_{1}\right) \rho_{2}} \int_{0}^{t} e^{4 \delta_{1} \tau} H_{1}(\tau) d \tau\right)^{1 / 2} e^{-2 \delta_{1} t}-\frac{6 \nu_{2}\left(C_{1}+C_{3}\right)}{\left(l_{2}-l_{1}\right)^{2}}\left(\int_{0}^{t} e^{4 \delta_{1} \tau} H(\tau) d \tau\right)^{1 / 4} e^{-\delta_{1} t}, \\
\left|f_{3}(t)\right| \leq\left(\frac{12}{5\left(l_{3}-l_{2}\right) \rho_{3}} \int_{0}^{t} e^{4 \delta_{1} \tau} H_{1}(\tau) d \tau\right)^{1 / 2} e^{-2 \delta_{1} t}-\frac{6 \nu_{3} C_{3}}{\left(l_{3}-l_{2}\right)^{2}}\left(\int_{0}^{t} e^{4 \delta_{1} \tau} H(\tau) d \tau\right)^{1 / 4} e^{-\delta_{1} t} .
\end{gathered}
$$

The functions $H(t)$ and $H_{1}(t)$ in (69) are estimated from above in (51) and (66). Therefore, in view of convergence of (39) and (60), together with $\left|a_{j}(y, t)\right|$ and $\left|w_{j}(y, t)\right|$, we also have $\left|f_{j}(t)\right| \rightarrow 0$ as $t \rightarrow \infty$.

\section{STATIONARY SOLUTION}

Let us write a stationary solution of the problem (21)-(25) $\left(a_{10}^{s}\right.$ and $a_{30}^{s}$ take constant values on the walls $y=0$ and $y=l_{3}$; hereafter, $a_{10}^{s} \neq 0$ ):

$$
\begin{gathered}
a_{1}^{s}(\xi)=a_{10}^{s}\left[A_{1} \xi+1\right], \quad 0<\xi=y / l_{1}<1, \\
a_{2}^{s}(\xi)=a_{10}^{s}\left[A_{1}\left((\xi-1) \bar{k}_{1}+1\right)+1\right], \quad 1<\xi<1 / \bar{l}_{1}, \\
a_{3}^{s}(\xi)=a_{10}^{s}\left[\bar{k}_{1} \bar{k}_{2} A_{1}\left(\xi-\bar{l}_{2} / \bar{l}_{1}\right)+a_{30}^{s} / a_{10}^{s}\right],
\end{gathered}
$$


and problem (15)-(20):

$$
\begin{gathered}
w_{1}^{s}(\xi)=\frac{l_{1} \varkappa_{2} a_{2}^{s}\left(l_{2}\right)}{\mu_{2}} \frac{\bar{l}_{1}\left(\bar{l}_{1}-1\right)}{m_{1}}\left(2 \xi-3 \xi^{2}\right)\left[\frac{\varkappa_{1} a_{1}^{s}\left(l_{1}\right)}{\varkappa_{2} a_{2}^{s}\left(l_{2}\right)}-\frac{m_{5}}{m_{4}}\left(\bar{l}_{1}-1\right)\right], \quad 0<\xi=y / l_{1}<1, \\
w_{2}^{s}(\xi)=-\frac{l_{1} \varkappa_{2} a_{2}^{s}\left(l_{2}\right)}{\mu_{2}}\left[\frac{3 \bar{l}_{1} m_{5}}{m_{4}}(\xi-1)^{2}+\frac{\bar{l}_{1}\left(\bar{l}_{1}-1\right)}{m_{1}}\left(1+4 \bar{\mu}_{1}(\xi-1)\right)\right. \\
\left.\times\left(\frac{\varkappa_{1} a_{1}^{s}\left(l_{1}\right)}{\varkappa_{2} a_{2}^{s}\left(l_{2}\right)}-\frac{m_{5}\left(\bar{l}_{1}-1\right)}{m_{4}}\right)+\frac{2 \varkappa_{1} a_{1}^{s}\left(l_{1}\right)}{\varkappa_{2} a_{2}^{s}\left(l_{2}\right)}(\xi-1)\right], \quad 1<\xi<1 / \bar{l}_{1}, \\
w_{3}^{s}(\xi)=\frac{2 \bar{\mu}_{2} l_{1} \varkappa_{2} a_{2}^{s}\left(l_{2}\right)}{\mu_{2}}\left(\xi-\frac{\bar{l}_{2}}{\bar{l}_{1}}\right)\left[-\frac{3 m_{6} \bar{l}_{1}}{4\left(\bar{l}_{2}-1\right)}\left(\xi+\frac{\bar{l}_{2}-2}{\bar{l}_{1}}\right)\right. \\
f_{1}^{s}=\frac{A_{2} \nu_{1}\left(\bar{l}_{1}-1\right)}{m_{1}}\left(\frac{\varkappa_{2} m_{5}\left(\bar{l}_{1}-1\right)}{m_{1} m_{4}}-\frac{\varkappa_{1} a_{1}^{s}\left(l_{1}\right)}{\varkappa_{1} \varkappa_{2} \varkappa_{2} a_{2}^{s}\left(l_{2}\right)}-1\right], \quad 1 / \bar{l}_{1}<\xi<\bar{l}_{2} / \bar{l}_{1}, \\
\left.m_{4}\left(\bar{l}_{1}-1\right)\right), \quad f_{2}^{s}=\frac{A_{2} \nu_{2} m_{5}}{m_{4}}, \quad f_{3}^{s}=\frac{A_{2} \nu_{3} \bar{\mu}_{2} m_{6}}{2\left(\bar{l}_{2}-1\right)},
\end{gathered}
$$

where

$$
\begin{gathered}
A_{1}=\bar{l}_{1} a_{30}^{s} /\left(a_{10}^{s}-1\right) g^{-1}, \quad A_{2}=6 \varkappa_{2} a_{2}^{s}\left(l_{2}\right) l_{2}^{-1} \mu_{2}^{-1}, \quad \bar{l}_{1}=l_{1} / l_{2}, \quad \bar{l}_{2}=l_{3} / l_{2}, \\
\bar{k}_{1}=k_{1} / k_{2}, \quad \bar{k}_{2}=k_{2} / k_{3}, \quad \bar{\mu}_{1}=\mu_{1} / \mu_{2}, \quad \bar{\mu}_{2}=\mu_{2} / \mu_{3},
\end{gathered}
$$

whereas the constants $g, m_{1}, m_{2}, m_{3}, m_{4}, m_{5}$, and $m_{6}$ are calculated according to the available formulas.

Introducing the differences

$$
\hat{a}_{j}(y, t)=a_{j}^{s}(y)-a_{j}(y, t), \quad \widehat{w}_{j}(y, t)=w_{j}^{s}(y)-w_{j}(y, t), \quad g(t)=f_{j}^{s}-f(t)
$$

and carrying out the calculations analogous to those in Section 2, we can prove that the solution of the nonstationary problem reaches the steady regime $a_{j}^{s}(y), w_{j}^{s}(y)$, and $f_{j}^{s}=$ const under the conditions of convergence of the second integrals in (39), (60) and the integral $\int_{0}^{\infty} e^{\delta \tau}\left|a_{k 0}^{s}-a_{k 0}(\tau)\right| d \tau$ for $k=1,3$. Let us note that, instead of (39) and (60), it suffices to require that

$$
\left|a_{k 0}^{s}-a_{k 0}(t)\right| \leq \alpha(t+\gamma)^{-n}
$$

with some constants $n>1, \alpha>0$, and $\gamma>0$.

Remark 4. Owing to the a priori estimates in Section 2, the solutions of (15)-(20) and (21)-(25) are classical:

$$
w_{j}(y, t), a_{j}(y, t) \in C^{2}\left(\Omega_{j}\right) \cap C^{1}(0, T], \quad \Omega_{1}=\left[0, l_{1}\right], \quad \Omega_{2}=\left[l_{1}, l_{2}\right], \quad \Omega_{3}=\left[l_{2}, l_{3}\right] .
$$

It is also easy to show the continuity of the derivatives $w_{j y}, w_{j y y}, a_{j y}$ and $a_{j y y}$. Using the above-obtained estimates for $w_{j t}, f_{j}$ and $a_{j t}$, the estimates for $w_{j y y}$ and $a_{j y y}$ can be found from (15) and (21); therefore, for example,

$$
\left|w_{j y}\left(y_{1}, t\right)-w_{j y}\left(y_{2}, t\right)\right|=\left|\int_{y_{1}}^{y_{2}} w_{j y y} d y\right| \leq\left|y_{1}-y_{2}\right| \max _{y \in \Omega_{j}, t \in(0, T]}\left|w_{j y y}\right|
$$

for all $y_{1}, y_{2} \in \Omega_{j}$. Hence, the functions $w_{j y}(y, t)$ are continuous on their domains, and the boundary conditions for the tangent stresses (19) are fulfilled in the sense of continuous functions. The estimates for $a_{j y}$ can be obtained by analogy.

\section{ACKNOWLEDGMENTS}

The authors were supported by the Russian Foundation for Basic Research (project no. 14-0100067). 


\section{REFERENCES}

1. V. K. Andreev, V. E. Zakhvataev, and E. A. Ryabitskii, Thermocapillary Instability (Nauka, Novosibirsk, 2000) [in Russian].

2. A. Nepomnyashii, I. Simanovskii, and J.-C. Legros, Interfacial Convection in Multilayer System (Springer, New York, 2006).

3. R. Narayanan and D. Schwabe, Interfacial Fluid Dynamics and Transport Processes (Springer, Berlin, 2003).

4. R. Kh. Zeytovnian, Convection in Fluids (Springer, Dordrecht, 2009).

5. V. K. Andreev, The Birikh Solution of the Convection Equations and Some Generalizations, Preprint No. 1-10 (Inst. Vychisl. Model., Krasnoyarsk, 2010).

6. V. K. Andreev, O. V. Kaptsov, V. V. Pukhnachov, and A. A. Rodionov, Applications of Group-Theoretical Methods in Hydrodynamics (Kluwer Acad. Publ., Dordrecht, 1998).

7. V. K. Andreev, Yu. A. Gaponenko, O. N. Goncharova, and V. V. Pukhnachov, Mathematical Models of Convection (Walter de Gruyter, Berlin, 2012).

8. L. G. Napolitano, "Plane Marangoni-Poiseuille Flow of Two Immiscible Fluids," Acta Astronautica 7 (4-5), $461-478(1980)$.

9. V. K. Andreev and V. B. Bekezhanova, Stability of Nonisothermal Fluids (Sibirsk. Federal. Univ., Krasnoyarsk, 2010) [in Russian].

10. V. K. Andreev, "On an Adjoint Initial-Boundary Value Problem," Differentsyal'nye Uravneniya 44 (12), 1667 1673 (2008) [Differential Equations 44 (12), 1730-1736 (2008)].

11. V. K. Andreev and V. B. Bekezhanova, "Stability of Nonisothermal Fluids," J. Appl. Mech. Techn. Phys. $54(2), 171-184(2013)$.

12. V. K. Andreev and E. N. Lemeshkova, "The Evolution of the Thermocapillary Motion of Three Fluids in a Plane Layer,” Prikl. Mat. Mekh. 78 (4), 485-492 (2014) [J. Appl. Math. Mech. 78 (4), 341-347 (2014)].

13. I. V. Denisova, "On the Problem of Thermocapillary Convection for Two Incompressible Fluids Separated by a Closed Interface," Progr. Nonlinear Differential Equations Appl. 61, 45-64 (2005).

14. I. V. Denisova, "Thermocapillary Convection Problem for Two Compressible Immiscible Fluids," Microgravity Sci. Technol. 20 (3-4), 287-291 (2008).

15. E. N. Lemeshkova, "About the Friedrichs Inequality for a Domain Consisted of Three Segments," in Some Actual Problems of Modern Mathematics and Mathematical Education: Herzen Readings. Proceedings of the Scientific Conference, April 11-16, 2012, St. Petersburg (ParkKom, St. Petersburg, 2011), pp. 80-84. 